Proceedings of the 50th Hawaii International Conference on System Sciences | 2017

\title{
Towards Open Smart Services Platform
}

\author{
Hamid R. Motahari Nezhad \\ IBM Almaden Research Center, \\ San Jose, CA, USA \\ motahari@us.ibm.com
}

\author{
Larisa Schwartz \\ IBM TJ Watson Research Center, \\ Yorktown, NY, USA \\ lshwart@us.ibm.com
}

\begin{abstract}
The landscape of services in the enterprise has changed significantly for both service providers and service clients over the last few years. In the IT services domain, the mega IT outsourcing service deals with a sole provider are diminishing fast. A typical service client is now consuming multiple IT services, from specialized providers, and services contracts has become smaller in size and duration. More importantly the line of business, not the IT, owns the decisions and the relationship for consuming services. This has also shifted the service consumption input from IT requirements into the business requirements. This new world is posing a new and unique set of opportunities and challenges for service providers in offering services, which include third party providers, to their clients, and for service clients to consume services from multiple providers. To facilitate offering and consuming such multi-vendor services, in this paper, we present a conceptual architecture for an open services platform which enables a given server provider (a service integrator) to offer services to its clients that are a mixture of its own and other services from third party providers. It also enables service clients to look for and choose services from multiple vendors in a seamless, integrated and consistent manner.
\end{abstract}

\section{Introduction}

A service is defined as the application of competence for the benefit of another entity [2]. In the business context, services are offered by a business to individuals or other businesses. In particular, IT services are offered as a combination of people and computational services in which an IT service provider offers to the business benefit of a client. The IT services landscape has been transformed considerably over the last decade. With the advent of cloud, SaaS (software as a service) and automation-based outsourcing, the traditional models of IT service offering and consumption has changed significantly. Businesses do not own their IT infrastructure and software as often. They have shifted to owning their business functions and operate it over externally provided software as a service, and externally sources services. Also in the enterprise, more and more, business lines, and not IT organization as it used to before, owns the decision to procure and consume external IT services [3]. An IDC survey [5] shows that in today's world over $60 \%$ of enterprise technology projects are funded by lines of business rather than IT. As a result, there are multiple service providers, typically offering services to the same client (referred as multi-sourcing [3]). Clients are in need of an integrated service experience, and this problem is now owned by the clients. At the service contract level, this translates into fewer large mega IT deals. Service providers are facing demands for smaller service contract size and duration. For instance, in 2013, the number of IT contracts has climbed $2 \%$, and the total contract value (TCV) has declined $18.2 \%$. Clients are increasingly engaging with best-of-bread providers, for each particular service, rather than a mega service provider that offers all their IT service needs. Therefore, multisourcing has become more pervasive.

These changes have posed a number of opportunities and challenges for the industry. From a service provider point of view, they do not own the whole client service operation, and have to work with a number of other service providers in the client environment. In the client's side, the role of $\mathrm{CIO}$ in an organization has transformed from an IT service management to a business-centric management of procured IT services [6]. And, the client (CIO) is left responsible for the integrated service experience across the enterprise. To this end, a new role in the IT services industry has emerged, referred to as service integrators. These are service providers in business of integrating services of a given client. This model is complex and not scalable for all clients, as not all services that need to be integrated are known apriori by service integrator, and they may change often. As another consequence of business lines owning the service consumption decisions is that the 
input for service procurement is transformed from purely IT requirements into business and IT requirements. All these highlights the need for dealing with the problem of service integration in a seempless, integrated and consistent manner including the alignment between business-level requirements and IT service provisioning.

As the services industry moves more and more towards multi-sourcing, we envision that the role of service integrators will be more prominent. However, service integrators will find offering a seamless and integrated service experience to their clients a major challenge due to the need for performing one-of-a-kind integration efforts, and lack of systematic methods to ensure a consistent service level experience to the enterprise clients..

In this paper, we argue for the need for an open services platform, which a service integrator may operate, and in which various service providers can be offered following a "plug and play" model. Such a platform may define a standardized, agile, and cooperative interaction framework for services providers to offer and deliver services to clients in a seamless manner. The platform should offer common services management and governance functionalities to support a smart services ecosystem starting from digesting the business requirements of clients, mapping them to IT (services) requirements, composing services in support of the business needs of the client and offer service guarantees and governance over a diverse set of services.

We present a conceptual architecture for the open services platform which supports a smart services system in which service providers are able to offer their services with functionality descriptions that allows the functional composition of various services to form larger business functions. The platform employs cognitive capabilities in digesting business requirements, and business function specifications of clients, and enables mapping of those into functional composition of IT services that achieves the business objects of the client. The rest of the paper is organized as follows. In Section 2, we characterize the problem and describe the requirements of an open services platform. Section 3 introduces the open services platform and provides a conceptual architecture for it. Related work is presented in Section 4, and we conclude and present future work in Section 5.

\section{Service Consumption: A Changing Market Landscape}

Traditionally, outsourcing services market is segmented into three main types of clients: cost cutters, growth seekers and innovators. While the clients in the first category looked at services acquisition as a vehicle for standardization and reducing cost, the growth seekers have taking strategic moves and partnership with service providers as a way to support the scalability and the growth of their business. The last group, i.e. innovators are usually looking for market leaders to transform a state of a business or a business model, and therefore look at potential service providers as a key partner in such a transformation. In this respect, the first category are after out of the box and standard offerings, while clients in the $2^{\text {nd }}$ and $3^{\text {rd }}$ category have been after customized deals to achieve their goals. Cloud services offerings (and specifically in form of software-as-aservice offerings, and services ecosystems around them) has provided not only standardization but allow access to agility and innovation. Most of the $2^{\text {nd }}$ category clients have shifted towards such service consumption models, according to a recent Gartner report [9]. This showcases the move towards more consolidation of service consumption by clients as cloud services.

While this move accelerates over the next few years, enterprises consume best of the breed services in each sector, supporting business functions and business processes. The new challenges will not only include management of multiple service providers at the client side [3]. To support this change in the service consumption model, we envision that in the near future, there will be large service integrators (or service brokers) that will take the responsibility of integrating services from multiple providers for clients.

If such a move materializes, there are new technical and business challenges for both service clients, and for service integrators to respond to the requirements of this new market, as described below.

Business-defined service sourcing and management: The service integrator and manager should offer the ability to source, manage, and deliver end-to-end services that solves a business problem. In deed, the service provider may need to offer a systematic method to map business-level solution requirements of the clients to concrete, IT functions (services) and potentially support hybrid sourcing strategies in which a combination of cloud and people services meet the requirements. In particular, in some application, the labor services may be offered through crowdsourced workforce in contrast to traditional inhouse sourcing to support acquired services.

Managing multi-vendors services with hybrid device, software, and infrastructure offerings: Hybrid and multi-vendor services in the IT landscape will be the new norm at every level of the stack for clients. Service management for all IT service sourcing and delivery for a client need to cover different types of services, potentially by diverse vendors, acting as a 
trusted broker and integrator for the clients. A critical challenge is the design of an (industry) ecosystem and methodology for smart services that support cooperative/coordinated service provider operation.

\section{The Open Smart Services Platform}

In this paper, we argue that with the proliferation of online and cloud-based service offerings in the IT

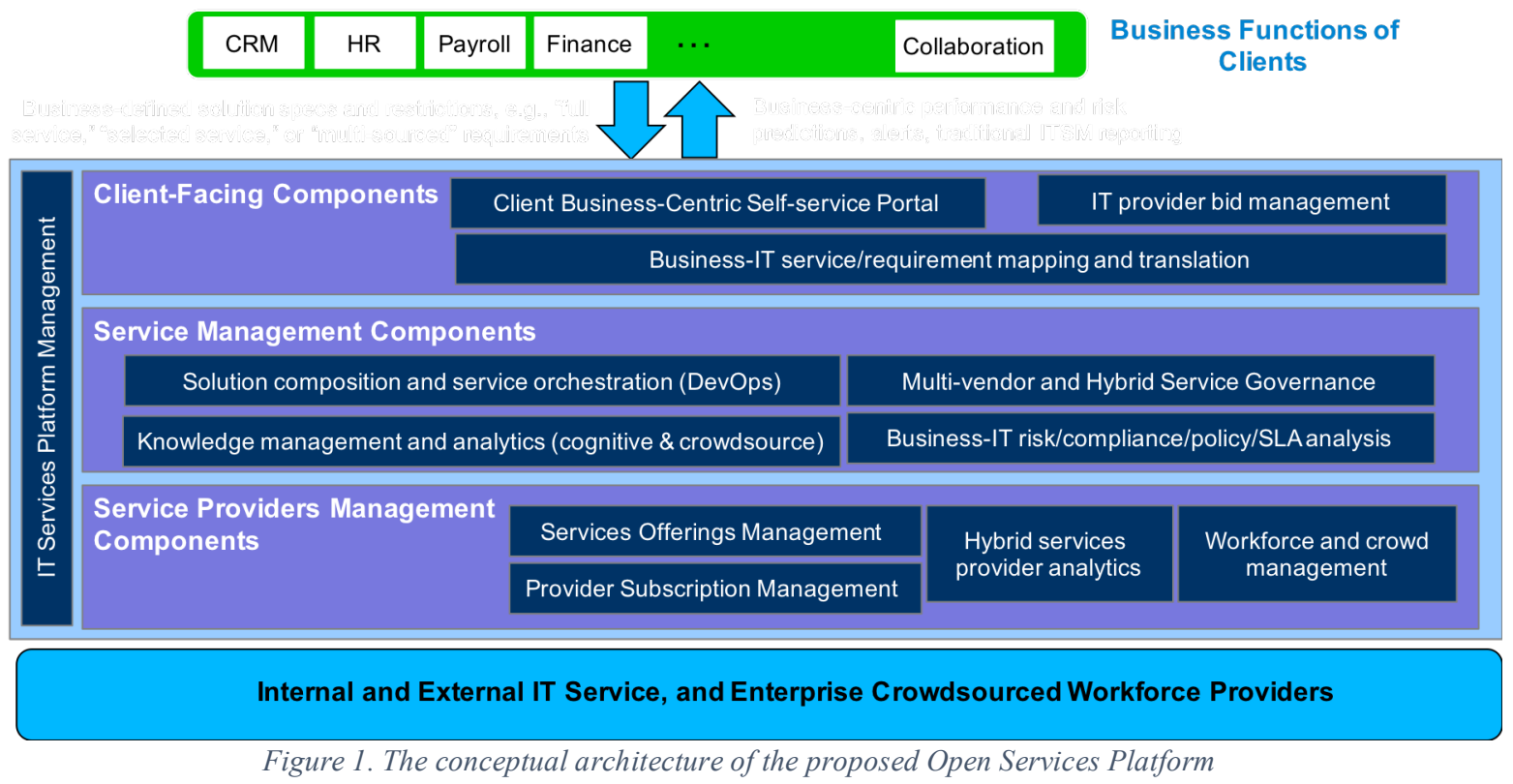

Cross-domain and multi-provider IT governance: In order for the service integrator or broker to be able to provide certain service levels in a consistent and continuous manner, new models for defining relationships, inter-dependencies, and risk assessment across service providers will be needed. Especially, it is important and challenging to define a governance model and function to interpret a clients' "business-level" implications from correlated IT activities/events/faults across multiple vendors' domains. New risk assessment and management, e.g., via implication prediction for services management, is an open salient challenge.

Self-service service catalogs, and provisioning and management: The eventual goal for a service broker is enabling a self-service model for service consumption. This is a challenging endeavor requiring knowledge-driven techniques and analysis to understand the current business practice of clients, the mappings into equivalent processes over the candidate service vendors, participating in the integration, and offering component business functions. Once such services are being consumed by clients, there is also the need to offer monitoring and consolidated reporting dashboards for key stakeholders in the business (and from different perspectives). services domain and their growing consumption by service clients, there is a need for an open services platform that enables service brokers to leverage various service providers to offer integrated services to clients, and service clients to use such open platforms as a source for finding, integrating and consuming offered services. The platforms should act as one stop shop, and offer a unified service experience for the clients to relieve each and every client from dealing with multiple vendors and integrating their services directly. For a service provider, a platform may offer service offering management functionalities, exposure to clients, and integration capabilities with other complementary offerings. As an analogy for an open services platform, consider App Stores as a platform for unifying the app discovery and consumption experience. Nevertheless, the App Stores are offering apps directly, however, they lack advanced brokerage functionalities that are needed in a business context for service offering and consumption.

Figure 1 presents the proposed conceptual architecture for an open services platform. The platform provides components to support offering and consumption of various types of IT services ranging from infrastructure, software, and people services. The platform may be a source for services covering various business functions needed to run a business (various industries), and also pure IT level services. The platform 
should enable sourcing desired business functions, in case they are offered in the platform, at a business level, going beyond the traditional IT service sourcing model. The keyword "smart" in the title of the platform refers to offering data and cognitive analytic functionalities, discussed below, to serve the service providers and consumers. The platform should provide a service offering framework and model that enable offering of smart services (i.e. services that are configurable, adaptive, context- and client- aware, intelligent, or autonomous, i.e., self-healing and self-recovery, potentially through leveraging multitude of interchangeable service providers).

In the following, we describe the key capabilities of the open smart services platform, depicted in Figure 1. The proposed architecture for the platform consists of three layers: (i) service provider management, (ii) service management, and (iii) clients facing.

Service provider management components. This layer caters components for enabling service providers to get onboard and be individually consumed and managed. In particular, the Services Offering Management component should provide a framework, methods and interfaces to allow services to be registered, described including information on the consumption of the services. The Provider Subscription Management component is responsible for management of subscription information of clients for using different service providers, and their SLA management [13]. It also should manage the subscription of service providers in the platform, offering service contract management.

The Provider Service Analytics components provides descriptive and predictive analytics on the information from past consumption of services, including SLA, subscription and usage statistics, cost and performance metrics. The other important components in this layer is the ability to offer noncomputational services, e.g., workforce and crowdsourced labor services with expertise in specific business functions or IT support services. The issues handled by this component is crowdsourcing in an enterprise context, and service management context to enable hiding the complexity of sourcing and offering a guaranteed service level as if the provided workforce are that of the client company. There are other open challenges related to knowledge sharing, and transfer among workforce and client organizations.

Service Management Components. The middle layer components are the core differentiator and unique functionalities that are needed by service integrators, and here by the open service platform, as its value proposition to offer its services to both service consumers and providers. The knowledge management and analytical components need to cater for building a knowledge model over existing services in the platform, and provide various foundational services to the other components. The Business-IT risk, compliance and SLA analytics act as a meta layer on how to guarantee certain service levels to its clients using a pool of underlying services. The governance component related to putting in place a component for policy, regulation and compliance requirements in a multi-vendor setting, which has not been studied in depth so far. And, the service composition and orchestration component should offer functionality for understanding how to compose services in a hybrid, multi-type services in a dynamic and as-needed basis, as opposed to today's static apriori-defined composition and orchestration logics.

Client facing components. The client facing components offer functionalities to support two key value propositions: (i) client self-service for clients that are looking for standard types of services for various business functions, (ii) elevating the expression of services from an IT style into business level requirements specification.

To support above scenario, there is a need for functionalities supporting mapping the business requirements into IT services that support each business functions, and make an assessment of the client requirements and whether those will be able to be met with existing services in the platform, or there is a need for special bid for custom services and do a cost/price analysis of different options. If the client chose to go the custom route (the growth seeking, and innovation seeking clients), there should be a component for managing client bids in the platform for hybrid people services for customization development over existing services, or in-house service offerings.

Cognitive service solution design. A key design principle for service composition, different than traditional notion of service composition in Web services domain [1], is that of cognitive composition of functionally described services, as opposed to prescribed as in case of web services. This is particularly different to traditional API-based service composition and design in the sense that in most cases that actual IT plumbing between services happen at the delivery time. What is important at the design time is understanding that the services that are composed are offering the functionalities of the interest by a client, and can meet its non-functional requirements. Existing service composition approaches define rigid structures for service definition, an approach which has failed to scale to the internet of services. We argue that there is a need for a cognitive service solution design approach that enables composition of service solutions at a functional-level, and in a flexible, progressive and dynamic manner in which the platform provides guidance on configuration, customizability and the 
fitness of potential services for the service requirements at hand.

Bid and proposal automation. For clients that need customized solution design based on services in the platform, a next research challenge is the automatic composition of proposal documents in response to client RFPs. In preparing such proposal documents, the system should be able to include assessment of the the proposal content (included solutions) in meeting the client requirements, and compliance with applicable laws, policies and regulations

Labor services. Another important trend in the enterprise services transformation is in the workforce composition of enterprises, specifically with the advent of enterprise-grade crowdsourced workforce platforms and providers. This has enabled the extreme vision of the enterprise of one, in which all but one (the owner) of a (small to medium) business are contingent and ondemand (and potentially online) staffers [7]. Indeed, the number of contingent workers in the enterprise has grown steadily since 1995 (150\%), and $80 \%$ of US companies deploy contingent workers. In particular, online staffing and crowdsourcing for enterprise staffing are trending (Online staffing grew 60\% in 2013, and to $\$ 3 \mathrm{~B}$ in 2014 [8]). And, the online and crowdsourced staff have been increasingly deployed in the IT services management domain. It remains a challenge to leverage crowdsourced workforce to offer enterprise-grade labor service experience.

In the following, we review how progress in some line of research brings us closer to the vision of an open smart services platform.

\subsection{Client Requirement to Services Mapping}

Client service requirements are often formulated and described in RFP (Request for Proposal) documents. The research challenge is understanding client requirements from these RFP documents, consisting of a set of unstructured documents written in natural language, and finding the set of services in the services platform matching the requirements. We have developed a cognitive Requirements Analytics Tool, called RFPCog [12] for requirements identifications and anlytics. RFPCog processes RFP documents including attachments to extract requirements from RFP documents, and digests and organizes the requirements in the RFP documents in terms of scope, requested IT services, service levels, and various services that clients are requesting.

To this end, we have extended RFPCog to map the discovered requirements to the description of available services in the open services platform. This is done based on matching the descriptions of the required IT service in the RFP to the functional description of services in the platform. This is based on the notion of matching individual requirements to services in the platform. This operation allows compiling the set of services from the platform that, together, fulfill IT service requirements described in a given RFP. Following this approach, we are able to identify gaps in the requirements (requirements that are not covered by available services), and potential mistmaches in the client's requirements with functionalities offered by enlisted services. In the platform, we index each of the available services against a service offering catalog. In particular, we have adopted ITIL (IT Infrastructure Library) service taxonomy to index the services in the platform for this purpose. This indexing is used, as an intermediary, during the matching of the requirements to services. Indeed, as often IT service requirement description from client is closer to ITIL language, the mapping of requirements to ITIL service taxonomy, and then to indexed services yields better results than direct matching of service descriptions to requirements.

\subsection{Business Function and Process Discovery and Composition}

One of the other functionalities of an open services platform is finding, representing and understanding the client's business. This entails offering the following functions in the services platform: (i) automated discovery of client business application and dependencies on client's IT components. And, (ii) automated understanding of client's business processes that are supported by those business applications in client's business functions. This involves understanding not only how each of the applications are used in the overall business processes. Such information may be captured in manual and process description documents.

In particular, the discovery domain of an enterprise needs digesting the unstructured but semantically linked information in the enterprise (for a given business function) of a client. In a prior work, we introduced the concept of Cognitive BPM [10], which refers to the use of cognitive computing technology in digesting, understanding, formalization and enactment of business processes from unstructured information sources, potentially across a set of services, in an enterprise. We have introduced methods for the use of cognitive computing and in particular natural language processing and processing of linked, unstructured data to understand textual description of processes in documents, and related information exchanged in the conversation of employees/workers. In particular, we have developed an intelligent/cognitive assistant, called eAssistant [11], that digests this unstructured 
information and identifies process steps, their ordering relationships, their ownerships, and relationships to systems and applications in the enterprise.

A next step, beyond learning process steps, there is a need for cognitive approaches for understanding policies, laws, rules, regulations related to business processes and business functions in the enterprise. Such information could support the sourcing decisions for external services in assessing existing standard services, or configuration or customization of services.

A related desired functionality for an open platform is the composition of processes from hybrid services in the platform including the crowdsourced workforce to perform part of the process. Such an approach should be adaptive to support addition, change or removal of services during enactment, or change/update the composition logic on the fly, as well.

\subsection{Multi-Vendor Support, Integration and Management}

In order to provide support for multi-vendor consumption of services, the platform should offer standard interfaces for publishing services, and for service management operations, e.g., for monitoring/logging, configuration verification, and other aspects of service management by the clients. As part of service publication process, there is a need for ensuring of qualification for participating in the platform in order to minimize and manage platform's risk. Also, to support operations such as composition, the platform may proactively look for cross-provider compatibility/support and offer pre-defined bundles with different service levels, cost, etc. And, one of the key criteria for the success of the platform is to support "agile" onboarding and service consumption (cutting the scenarios from months to days, and to instantly for IT services) for operations such as join, leave, and replacement of service providers. The platform may also perform and enable clients to feed into continuous rating of service provider quality. Such criteria may be incorporated into provider selections, and in increasing trust in using services in the platform.

\subsection{Labor Workforce Transformation}

As mentioned earlier, a general trend in many service clients is the use and leverage of on-demand workforce to accomplish services, i.e., specialized workforce that are contracted, via the platform, in an asneeded basis (following the notion of the elasticity of resources). We envision the integration of enterprise crowdsourcing platforms with a pool of workforce with expertise in various services that are offered in the platform. Such workforce should be made available as services, and the platform should provide support for managing the expression of requirements, consumption of such services and managing service levels across workforce in a transparent and integrated manner. In an enterprise context, issues such as managing the workforce service brand, reputation, tacit knowledge transfer and incentives become important among workers.

In order to support the above vision, the platform should offer a abstractions and tools for a hybrid crowdcloud model (computing unit plus crowd unit for service delivery). These should enable integration and enactment of computational and people services. It should support integrating the results from computational units with crowd (on-demand employees) in a holistic and intelligent manner. In summary, we envision a workforce transformation in many service delivery scenarios in which more automation (intelligent agents and services) will be supplemented with a combination of employees and ondemand workforce. Such a new environment could be called hybrid IT services management practice.

\section{Related Work}

The problem of offering and consuming services has been studied in the context of computational (Web) services [1], as well in the context of people (enterprise) services [2]. However, the proliferation of services, in forms of software as a service, and the move towards on-demand workforce has created new opportunities and challenges for service delivery and consumptions for both service providers and service clients. And, the multi-sourcing trend by clients and the need for service integration [3] at the client side has created issues for both service clients and service providers. These transformations have created a new environment in which there is a need for an open services platform in which hybrid services are offered, and the consumption of business services, and not only IT services, and people services in a holistic and integrated manner are supported.

To deal with the problem of hybrid service integration for clients with multi-sourcing issue, a set of processes and guidelines called SIAM (service integration and management) has been authored by the UK government [4]. There are large and small service providers that have adopted SIAM's principles and processes to support clients in their service integration. However, we believe that with the growth in this space, one or more global service integrators will emerge to play this role in a larger scale. And, such a service integrator needs to be also a service broker. The notion 
of open smart services platform and associated conceptual architecture in this paper is presented as a proposal for such a global service broker and integrator.

A unique and novel requirement of the development of such an open and smart services platform is ability to support a myriad of hybrid services, and to support flexible and unstructured representation, specification, composition and consumption of such services. We believe that cognitive computing has the potential to support such such requirements. In particular, in this paper, we highlighted key advances in the cognitive technology in our work in support of the realization of the vision of open smart services platform. In particular, the work of cognitive BPM [10], and eAssistant [11] pave the way for understanding and learning business functions and business processes in an enterprise, the RFPCog [12] facilitates the digestion of the client requirement stated in natural language documents and specifications. These systems provide the initial building blocks for a cognitive open services platform.

\section{Conclusion and Future Work}

In this paper, we presented the notion of an open smart services platform, and its conceptual framework. The open services platform enables different service providers to offer various types of services including infrastructure, software and people services. The platform is the single point of contact for a provider consuming such services at the business level. We also reviewed the current progress towards realization of the vision and the architecture of an open smart services platform. To the best of our knowledge, this is the first paper describing this notion, and its corresponding architecture and implementation aspects.

As future work, we plan to investigate the realization of the components of the presented architecture to support offering smart services, and smart consumption of services by the client, including but not limited to, the composition of smart services, cognitive mapping of business requirements to IT services, and flexible and dynamic enactment of hybrid services to support the business of service clients in a holistic and transparent manner.

\section{References}

[1] Gustavo Alonso, Fabio Casati, Harumi Kuno, and Vijay Machiraju. Web Services: Concepts, Architectures and Applications (1st ed.). Springer, 2004.

[2] Spohrer, J., Maglio, P. P., Bailey, J. \& Gruhl, D. (2007). Steps toward a science of service systems. Computer, 40, 7177.

[3] Jamie Erbes, Hamid R. Motahari Nezhad, Sven Graupner: The Future of Enterprise IT in the Cloud. IEEE Computer 45(5): 66-72 (2012)

[4] SIAM Future Model Approach - Government Procurement Service - $\quad$ https://www.gov.uk/servicemanual/technology/service-integration.html, visited June 2016.

[5] "IDC Survey Reveals More than $60 \%$ of Enterprise Technology Projects Funded by Business Rather Than IT," http://www.idc.com/getdoc.jsp?containerId=prUS24370613, IDC 2013.

[6] "The strange life, death and rebirth of the CIO and what it means for the future of IT," ZDNet.com (http://www.zdnet.com/the-strange-life-death-and-rebirth-ofthe-cio-and-what-it-means-for-the-future-of-it-7000022113)/, 2013.

[7] Accenture Technology Vision 2014, Trend 2: From workforce to crowdsource: The rise of the borderless enterprise, http://www.accenture.com/microsite/ittechnology-trends-

2014/Documents/TechVision/Downloads/Accenture Techno logy Vision 2014 Trend2.pdf

[8] LionBridge, "The Crowd in the Cloud: Exploring the Future of Outsourcing," January 2013.

[9] Gartner, "Gartner Survey Reveals That SaaS Deployments Are Now Mission Critical" , http://www.gartner.com/newsroom/id/2923217, Nov 2014, accessed June 2016.

[10] Hamid R. Motahari Nezhad, Rama Akkiraju:

Towards Cognitive BPM as the Next Generation BPM Platform for Analytics-Driven Business Processes. Business Process Management Workshops 2014: 158-164

[11] Hamid Reza Motahari Nezhad,Cognitive Assistance at Work, 2015 AAAI Fall Symposium Series, AAAI Publications. November 2015.

[12] Hamid R. Motahari-Nezhad, Juan M. Cappi, Taiga Nakamurra, $\mathrm{Mu}$ Qiao, RFPCog: Linguistic-based Identification and Mapping of Service Requirements in Request for Proposals (RFPs) to IT Service Solutions, 49th Hawaii International Conference on System Sciences, IEEE, 2016.

[13] Mohamed Mohamed, Obinna Anya, Takashi Sakairi, Samir Tata, NagaPramod Mandagere, Heiko Ludwig: The rSLA Framework: Monitoring and Enforcement of Service Level Agreements for Cloud Services. SCC 2016: 625-632 